\title{
Infectious disease expert Michael S. Diamond wins the 2019 ASCI/Stanley J. Korsmeyer prize
}

$T_{\text {t }}$ he 2019 Stanley J. Korsmeyer award, given by the American Society for Clinical Investigation (ASCI), honors Michael S. Diamond of the Washington University School of Medicine for his work uncovering the molecular basis of disease caused by globally emerging RNA viruses. The annual prize, named for its first recipient, Stanley J. Korsmeyer, recognizes outstanding accomplishments in research and mentorship by ASCI members. Dr. Diamond (Figure 1) has made seminal contributions to our understanding of the pathogenesis of several emerging viruses, including the Dengue, West Nile, Chikungunya, and Zika viruses, as well as the host immune system responses to infection. The JCI recently spoke with Diamond about his remarkable research career.

JCI: Your bachelor's degree was in political science. What led you to enroll in an MD PhD program?

Diamond: I was interested in global politics, but I had attended a science high school in New York. In the back of my mind, I was also interested in science. Through fortuitous circumstances, I ended up participating in a research project in a laboratory at Columbia Presbyterian my senior year. That experience distilled for me that I really loved science and experimentation and wanted that to be my career track rather than going into international politics. Although my mentor in the lab was PhD-trained, there were many members of the department who strongly encouraged me to pursue a combined MD PhD program. They thought it would provide greater flexibility in science and potentially help with funding initiatives.

JCI: What influenced you to specialize in infectious disease?

Diamond: That decision stems from my interest in what started as global politics and then became global health. The idea that I would study something that would be affecting populations around the world was of great interest. I hoped I might be able to discover something that would impact not just the developed world but also the developing world. Although my $\mathrm{PhD}$ is in a program of cellular and developmental biology, my lab was really a basic immunology laboratory. Understanding the immune system in the context of pathogens also influenced my decision to pursue infectious diseases. That said, it was not a linear route that got me there, but it was always sort of percolating in the back of my mind that I wanted to shape my career around studying the basic science of problems related to global health. After I did my internship residency and ultimately did a fellowship, I figured out how I would combine these interests.

JCI: Can you tell us about your early work on Dengue virus?

Diamond: This work was done in Eva Harris' lab just as she was starting her laboratory. I met Eva through Julie Gerberding, who at the time was a was a faculty member at UCSF and later went on to be director of the CDC. Julie recommended that I talk to Eva because after doing my infectious disease clinical fellowship, I was interested in studying the immune basis of some pathogens in the context of global health. I did not want to work on HIV, because San Francisco was the epicenter of HIV, and there was a number of very prominent investigators there. As a person who was thinking about his career going forward, I thought it would be great to work on something that not everybody was working on, yet still could have a significant impact on global health. In Eva's lab, I worked, primarily in cell culture-based models, on understanding how innate immunity controlled Dengue virus and how Dengue virus could in theory evade some of those responses.

JCI: Once you set up your own lab, how did your work shift to focus on West Nile virus?

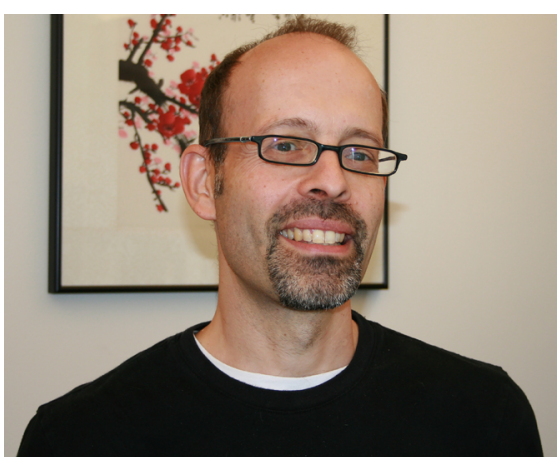

Figure 1. Michael S. Diamond is the recipient of the 2019 ASCl/Stanley J. Korsmeyer award.

Diamond: I came to Washington University in 2001. I was interviewing in about 2000, and, of course, in 1999, West Nile had just been entered into the United States in New York. There was a faculty member who was at UC Davis at the time (now in Albany), Laura Kramer, who collaborated with Eva Harris. Kramer, who is a mosquito specialist, and I had a conversation about what we thought might happen with West Nile virus. The idea that these vectors were present in the United States made it reasonable in her mind, and became so in my mind, that West Nile virus would spread and not be extinguished immediately. I thought it would be important to distinguish myself from Eva, who was already emerging as a significant leader in the Dengue field. West Nile was emerging, so the switch to a related virus was not a massive leap. I got a huge commitment when I came to Wash U from others (e.g., Skip Virgin, Dan Goldberg, and Ken Polonsky) that were willing help me build a biosafety level three facility to study West Nile virus and develop models. There really weren't that many facilities at the time, especially prior to $9 / 11$, and this allowed me to undertake those projects. There was a lot of support from medicine, immunology, as well as microbiology departments, in thinking about how to set up models of West Nile virus and studying the virus and host. I really wanted to use contemporary genetics to begin to understand host immunity to flaviviruses and to West Nile in particular. 
One of the reasons I came to Wash U was because of its strength in immunology in small animal models.

JCI: More recently, your lab adapted to focus on Zika virus. What enabled your group to develop the mouse model of Zika infection so quickly?

Diamond: People often ask me whether I have some sort of crystal ball to figure out what viruses should we be working on. The reality is, no. We work on a lot of different viruses - some of them have not emerged, but we still work on them, and some of them do emerge. That said, Zika was relatively unique in circumstance, and it traces back to an NIH meeting in 2015 on Chikungunya virus. During a coffee break, there were a couple of investigators from Brazil who mentioned to me that they were seeing unusual clinical phenotypes associated with what they thought was Zika virus infection. They were not sure at the time, but it seemed that there were babies being born with microcephaly to Zika-infected mothers. I had obtained Zika virus in the lab as part of a collection of flaviviruses that we use as comparative flaviviruses to study. This conversation was enough for me to talk to a postdoctoral fellow in my laboratory at the time, Helen Lazear, who now has her own lab at UNC, about initiating something. I basically said, "Why don't we start to learn more about Zika virus, figure out ways to grow and measure it, and develop an animal model with some of the phenotypes being seen in Brazil." Helen and postbaccalaureate student Derek Platt started working on this in June of 2015, and then as the epidemic expanded, we accelerated our work, with Jonathan Miner, another former postdoc and now faculty member, joining in the project. I think, because of that, we were many months ahead of the other groups in setting this up. We also had a lot of tools from our studies of other flaviviruses to test out what might be the best models to go forward with and how to refine them. The moral of the story to me is to pay attention. I have contacts out in different parts of the globe, and if there are viruses that are coming up, even if they haven't emerged yet, there's utility in developing some tools and reagents, which then will allow you to accelerate very quickly.
JCI: Do you think that there are things that the scientific community as a whole can do to better prepare for the next outbreak?

Diamond: That's a complex question. Obviously, understanding how families of viruses work in general is important, and that's basic research. Lynn Enquist had a symposium a few years ago, which in part focused on why our collective response to Zika was so fast compared to some other viruses. As a field, we really accelerated development of countermeasures, vaccines, and clinical trials by many, many different groups, and were able to really understand the biology. Part of it is because we understood the other viruses in the family - it's related to Dengue, it's related to West Nile. It was decades of work on those viruses that allowed us to really rapidly make progress, even in the setting of some rather unique features to Zika, such as vertical transmission and sexual transmission. Having a foundation in basic science, in pathogenesis of viruses, will help us be more prepared for whatever comes next, because in all likelihood the next emerging virus will be related to something. The question is, will it be an understudied area or not?

The second thing that really accelerated Zika work has to do with how we categorized viruses. Zika was classified as a biosafety level two virus. West Nile is a biosafety level three virus. Ebola is a biosafety level four virus. And what that means, besides the fact of the different protective gear that you need to take to work with it, is that only certain labs can work on biosafety level four. There are only a few around the country. The fact that Zika was classified as level two means that almost every lab and every institution in the United States (and other countries) could actually work with it, and so that enabled a huge global response by people. How you classify a virus actually matters in how many people will be able to do research.

JCI: If you reflect back on your own training, in what ways did your mentors shape your outlook on science?

Diamond: The person that most profoundly shaped the way I approach science, I think, was my $\mathrm{PhD}$ advisor Tim Springer at Harvard Medical School. I spent the most time with him, in terms of years that
I was actually training. Tim could be a little tough, people might say, and I think it's probably true. But he was tough because he was rigorous in performing, analyzing, and ultimately, publishing experiments. And I think I hold my students and trainees to that same level of rigor. As a mentor, I try to combine rigor with inspiring students to tackle challenging scientific questions. Eva Harris has a magical way of being excited about the science and motivating and inspiring people, and that energy was something that was really important to be able to bring to my lab. I have learned from my mentors the importance of respecting people's opinions, encouraging people to do things that maybe are a little bit off the beaten path, and allowing people to have latitude within some framework and being able to encourage them to try different things. Each level of my training provided me with a different component to the way that I try to run my laboratory. After my trainees leave my lab, I encourage them to find the position that they think will make them the most fulfilled. It's certainly rewarding to see both my graduate students as well as my postdocs get faculty positions, but I'm also excited for those that go into industry and have been doing really well in drug development.

JCI: What does winning the ASCI's Korsmeyer award mean to you?

Diamond: One of the things that I didn't realize until I won the award was how many people knew Stan here. Stan was at Wash $\mathrm{U}$ before he went to Harvard, and he had a terrific career, and made some fundamental discoveries in cell death and apoptosis. Unfortunately, his career was cut short by his illness. But it was remarkable how many people here have come up to me, faculty that I knew and faculty that I didn't know, and told me what a great honor this was and how terrific a person and scientist Stan was. For me, it's always nice to get an accolade, but I think it's especially nice to me that Stan spent a significant part of his career here. I'm also humbled because very few people get this award, and the past recipients are such superlative scientists. To be in their company is extraordinarily flattering.

\section{Sarah Jackson}

